University of New Hampshire

University of New Hampshire Scholars' Repository

Space Science Center

Institute for the Study of Earth, Oceans, and

Space (EOS)

$5-11-2009$

\title{
A compton telescope for remote location and identification of radioactive material
}

\author{
Peter F. Bloser \\ University of New Hampshire, Peter.Bloser@unh.edu \\ Jason S. Legere \\ University of New Hampshire, jslegere@unh.edu \\ Shirley M. Dame \\ University of New Hampshire - Main Campus \\ Mark L. McConnell \\ University of New Hampshire - Main Campus, mark.mcconnell@unh.edu \\ U Bravar \\ University of New Hampshire - Main Campus
}

See next page for additional authors

Follow this and additional works at: https://scholars.unh.edu/ssc

Part of the Astrophysics and Astronomy Commons

\section{Recommended Citation}

Bloser, P.F.; Legere, J.S.; Dame, S.M.; McConnell, M.L.; Bravar, U.; Ryan, J.M., "A compton telescope for remote location and identification of radioactive material," Technologies for Homeland Security, 2009. HST '09. IEEE Conference on , vol., no., pp.302,307, 11-12 May 2009 doi: 10.1109/THS.2009.5168050

This Conference Proceeding is brought to you for free and open access by the Institute for the Study of Earth, Oceans, and Space (EOS) at University of New Hampshire Scholars' Repository. It has been accepted for inclusion in Space Science Center by an authorized administrator of University of New Hampshire Scholars' Repository. For more information, please contact Scholarly.Communication@unh.edu. 


\section{Authors}

Peter F. Bloser, Jason S. Legere, Shirley M. Dame, Mark L. McConnell, U Bravar, and James M. Ryan 


\title{
A Compton Telescope for Remote Location and Identification of Radioactive Material
}

\author{
Peter F. Bloser, Jason S. Legere, Shirley M. Dame, Mark L. McConnell, Ulisse Bravar, James M. Ryan, \\ Space Science Center, University of New Hampshire, Durham, NH 03824, peter.bloser@unh.edu, jslegere@unh.edu, \\ smy67@,cisunix.unh.edu, mark.mcconnell@unh.edu, ulisse.bravar@unh.edu, james.ryan@unh.edu
}

\begin{abstract}
The spare detectors from NASA's Compton Gamma-Ray Observatory COMPTEL instrument have been reconfigured to demonstrate the capability at ground level to remotely locate and identify sources of gamma radiation. The Gamma-Ray Experimental Telescope Assembly (GRETA) employs two $28 \mathrm{~cm}$ diameter scintillation detectors separated by $95 \mathrm{~cm}$ : one $8.5 \mathrm{~cm}$ thick liquid scintillator detector and one $7.5 \mathrm{~cm}$ thick $\mathrm{NaI}(\mathrm{Tl})$ detector. The assembly electronics and real-time data acquisition system measures the energy deposits and time-of-flight for each coincident detection and compiles histograms of total energy and incident angle as computed using the kinematics of Compton scattering. GRETA's field of view is a cone with full angle approximately $120^{\circ}$. The sensitive energy range is 0.3 to $2.6 \mathrm{MeV}$. Energy resolution is $\sim 10 \%$ FWHM. The angular resolution is better than $5^{\circ}$.
\end{abstract}

We have previously reported measurements using a simplified readout configuration that limited GRETA's imaging ability to a rough $\left(\sim 15^{\circ}\right)$ directional sensitivity. Here we report on measurements using the full (15-channel) readout that can achieve $\sim 1.5-\mathrm{cm}$ position resolution in each scintillator and permits true Compton imaging using COMPTEL software. GRETA has been refurbished to allow for ease of transport and field use with updated real time imaging and identification software. We present most recent laboratory measurements of radioactive sources of concern, ${ }^{137} \mathrm{Cs}$ and ${ }^{60} \mathrm{Co}$, potential candidates used with radiological dispersal devices.

\section{INTRODUCTION}

\section{Motivation}

The remote detection and location of radioactive threat materials, specifically the key isotopes likely used in radiological dispersal devices (RDDs), is a serious problem facing national security [1]. RDDs, or dirty bombs, can be assembled in any number of configurations, with the function of dispersing radiological materials over populated areas. Most likely candidate materials are industrial radionuclides, ${ }^{137} \mathrm{Cs}$ or ${ }^{60} \mathrm{Co}$, that are widely used in a number of unsecured locations.
Nuclear material detection applications range from radiological screening at borders, detection of materials in transit, search and emergency response, and consequence and management recovery [1]. Searching for dangerous suspected quantities of radioactive material (RAM) is a difficult activity that can stall or suspend operations. Important detector properties include; it must be efficient at locating and identify RAM with minimal false alarm rates, rugged and mobile, and easy to operate. Standard equipment used to search for RAM includes hand held counters and spectrometers. Either device registers an increased count rate in the presence of RAM. The spectrometer can also identify the nature of the source, e.g., whether it is ${ }^{137} \mathrm{Cs}$ or ${ }^{60} \mathrm{Co}$. However, both devices must overcome the effect of background radiation and neither pinpoints the source other than by proximity inducing the high-count rate.

\section{Technique}

A better way would be to sense the presence of RAM from a safe standoff distance. Better still would be to locate the source and identify the isotope. To do this one must have an imaging $\gamma$-ray telescope that spectroscopically analyzes the imaged source. Such a device has already been used in space to image cosmic $\gamma$-ray sources in harsh, high background environments. Compton telescopes are the instrument of choice for $\gamma$-ray sources in the $1 \mathrm{MeV}$ range. Other techniques for producing $\gamma$-ray imaging exist but do not have the sensitivity of Compton telescopes.

For example, coded aperture imaging relies on the ability of the mask to absorb the incident photons (Figure 1.) At higher energies, this requires thicker masks that are impractical. This, coupled with the intrinsic background of the imaging system, renders coded aperture imaging less attractive at higher energies, i.e., $1 \mathrm{MeV}$.

A more effective approach at higher energies takes full advantage of the Compton effect, in which a photon scatters off an electron. Compton imaging becomes effective at energies above about $500 \mathrm{keV}$. The Compton telescope concept relies on measurements of both the scattered electron and the scattered photon. This double scatter approach typically relies on two separate detectors, one designed to measure the scattered photon and the location of the scatter interaction and the other detector designed to absorb the scattered photon to record its total energy and scatter direction.

Compton telescopes that measure only the energy of the scattered electron do not have the ability to determine the 
precise direction of the incident photon. Rather, the direction of the incoming photon is determined to lie along a so-called "event circle," whose parameters are defined by the kinematics of the scattering process. A large number of event circles are used to generate maps of the $\gamma$-ray emission within the field-of-view. Shown in Figure 2, from COMPTEL data [2], a cosmic $\gamma$-ray burst was localized with of order 100 detected photons. Figure 3 shows the COMPTEL instrument $[3,4]$ that flew in earth orbit for nine years as part of the payload on the Compton Gamma Ray Observatory (1991-2000). This instrument, operating between $750 \mathrm{keV}$ and $30 \mathrm{MeV}$, achieved a moderate angular resolution $\left(1^{\circ}-2^{\circ}\right)$ over a fairly large field-of-view.

One advantage of Compton imaging over coded aperture imaging is that the Compton imaging treats each photon individually. This means that background rejection becomes more efficient. On COMPTEL, time-of-flight information (measuring the time between the Compton scatter interaction and the subsequent absorption of the scattered photon) was employed to dramatically reduce the background from other sources. This is especially important for astronomical applications where orbiting spacecraft are subjected to a high level of both particle and photon backgrounds. We found that capability to also be practical for terrestrial applications, i.e., rejecting background $\gamma$-rays from outside the intended field of view.

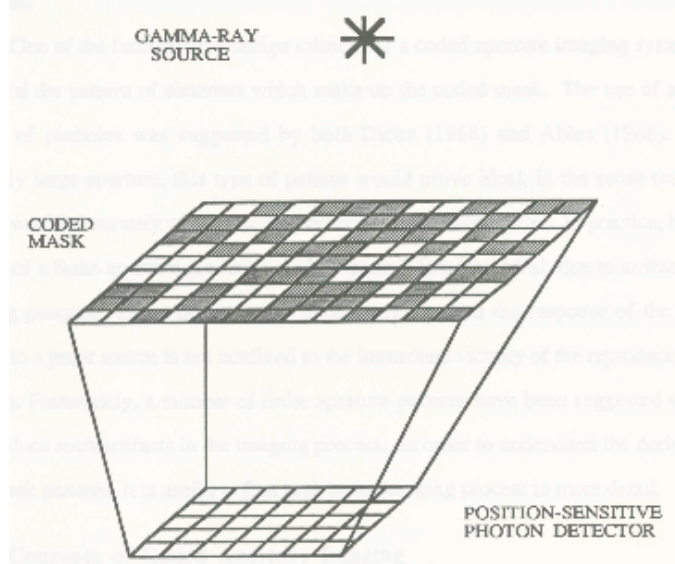

Figure 1 - Coded aperture concept using a coded mask with opaque elements and a position-sensitive photon detection plane that measures the resulting shadow cast by the $\gamma$-ray source.

\section{INSTRUMENT DESCRIPTION}

\section{The GRETA Instrument}

The Gamma-Ray Experimental Telescope Assembly (GRETA) employs two rugged cylindrical scintillation detector assemblies aligned on their cylindrical axes and separated by $95 \mathrm{~cm}$ (Figure 4). These detector assemblies are flight spares from the NASA Compton Gamma-Ray Observatory COMPTEL instrument.

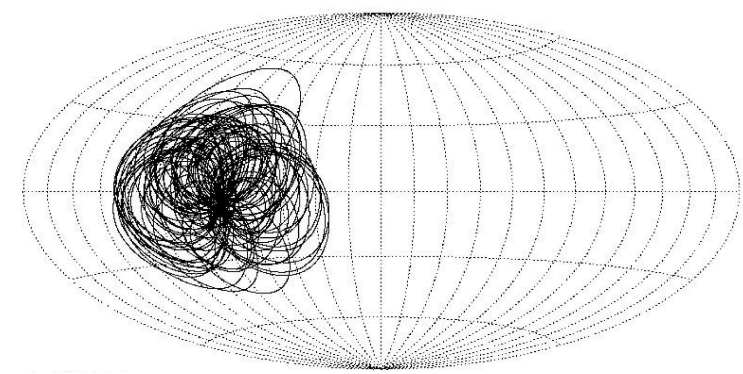

Figure 2 - Intersecting event annuli from the cosmic $\gamma$-ray burst of 1991 May 3.

The front detector (D1, Figure 4, top left) is an aluminum tank containing approximately 5.6 liters of liquid scintillator NE213A. The liquid scintillator tank features low-density aluminum honeycomb front and back cover plates, eight quartz glass windows distributed evenly around the circumference, an integrated metal bellows expansion reservoir and redundant seals to safely contain the liquid. Eight $51 \mathrm{~mm}$ diameter fast bialkali photomultiplier tubes (PMT), optically coupled to the eight windows view the interior volume. The interior walls are coated with white,

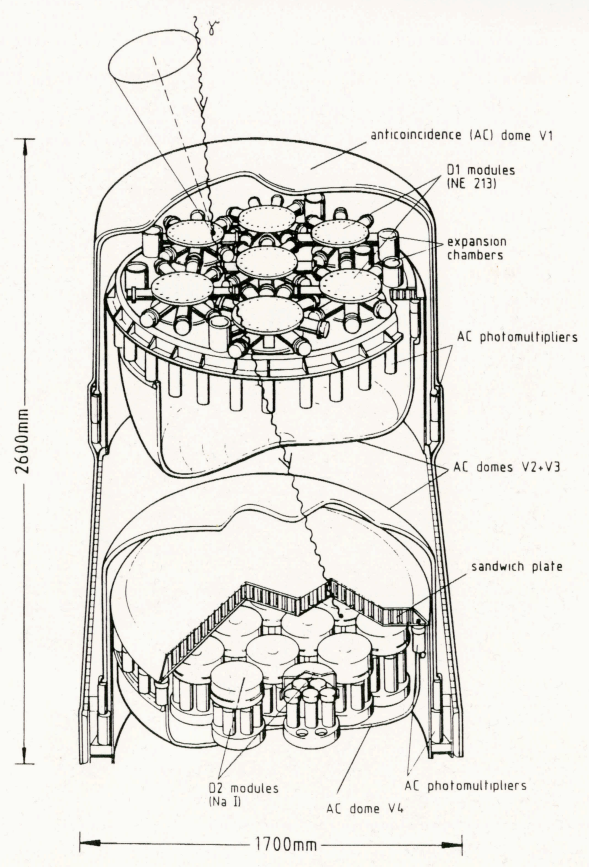

Figure 3 - A schematic of the COMPTEL instrument that was flown on the NASA Compton Gamma Ray Observatory (CGRO). The principle measurement is shown, along with the event circle that is reconstructed from the measured kinematics. The photons incident direction is known to have come from somewhere along the mantle of an event cone.

diffuse, reflector paint. The active liquid scintillator volume for D1 is $8.5 \mathrm{~cm}$ thick and $28 \mathrm{~cm}$ in diameter. The liquid scintillator, a hazardous material, would be replaced with plastic scintillator for future more transportable units. 


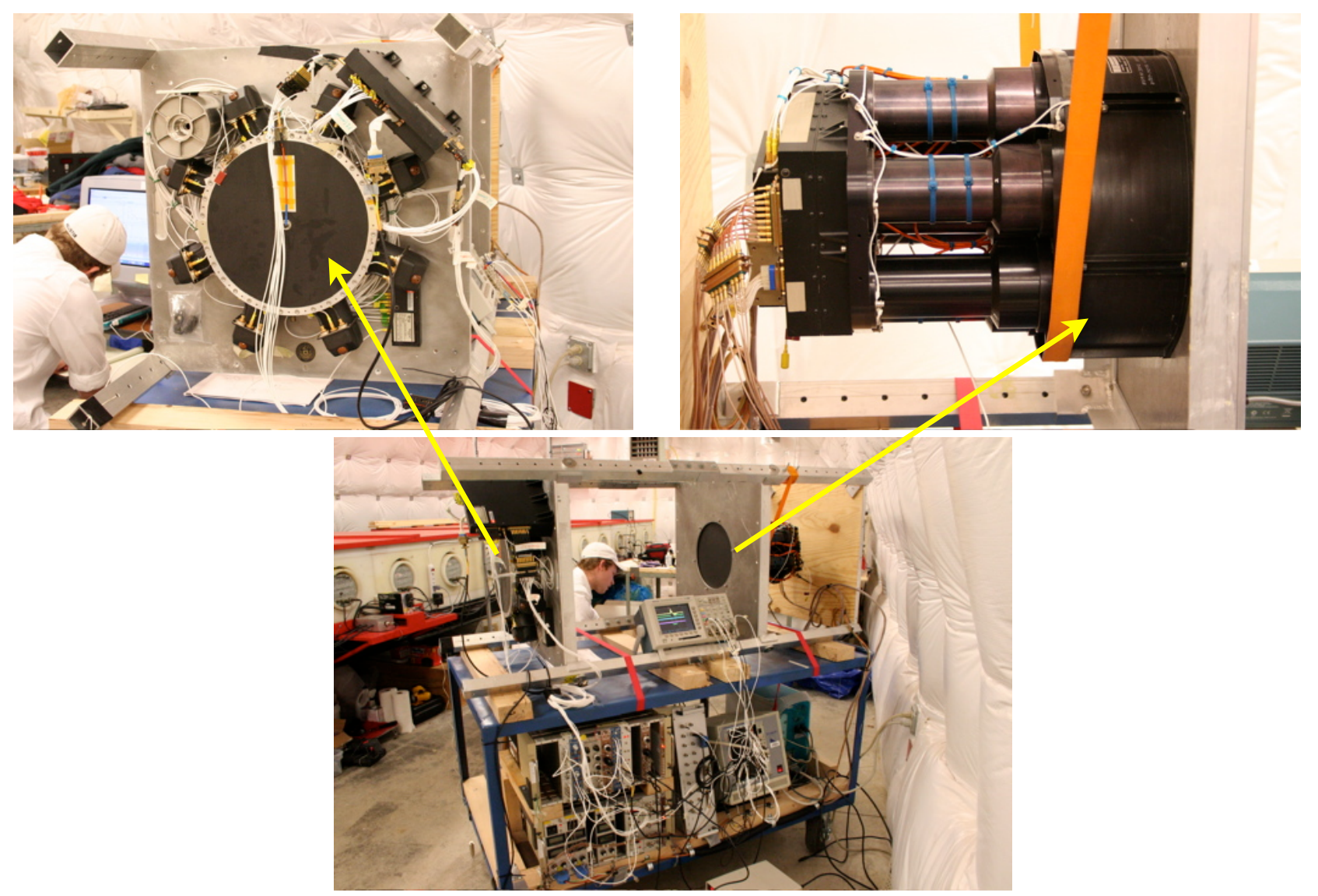

Figure 4 - D1 detector (top left), $28 \mathrm{~cm}$ diameter, $8.5 \mathrm{~cm}$ thick liquid scintillator tank viewed radially by eight $51 \mathrm{~mm}$ PMTs. D2 detector (top right), $28 \mathrm{~cm}$ diameter, $7.5 \mathrm{~cm}$ thick NaI(T1) scintillator viewed by seven $76 \mathrm{~mm}$ PMTs. The mobile GRETA system is shown with associated electronics on lower cart shelf (bottom center). Operator interface (laptop) not shown.

The rear detector (D2, Figure 4, top right) employs a $7.5 \mathrm{~cm}$ thick $28 \mathrm{~cm}$ diameter $\mathrm{NaI}(\mathrm{Tl})$ scintillator and seven $76 \mathrm{~mm}$ diameter fast bialkali PMTs that view the interior volume from the rear face. The D2 assembly features a low-density entrance window of honeycomb aluminum construction and a rugged hermetically sealed housing to protect the scintillator crystal and PMTs. The D1 and D2 detector materials and dimensions were selected to optimize detection via the Compton scattering process in the energy range $>300 \mathrm{keV}$. The GRETA electronics were more simply configured to ensure adequate response in the limited energy range of 0.4 to $3 \mathrm{MeV}$, the important range for RAM. However, active interrogation of high explosives need an energy range extending to at least $10 \mathrm{MeV}$, requiring flight-like electronics.

The front-end electronics for D1 and D2 are located on the detector assemblies. An aluminum frame aligns and supports the detector assemblies. The Compton telescope rests atop a rolling cart that also supports the power supplies and data acquisition electronics (Figure 4, bottom center). This configuration facilitated the field measurements reported here.
The D1 and D2 front-end electronics processes all PMT signals. Output signals are as follows.

- Pulse height signals, $(0.5 \mu \mathrm{s}$ shaping) for each PMT; 8 for D1, 7 for D2

- $\quad$ Sum pulse height signal, D1 and D2 $(0.5 \mu \mathrm{s}$ shaping)

- Constant fraction discriminator (CFD) timing signal; one each for D1 and D2

- $\quad$ Pulse shape discrimination (PSD) signal for D1 only; neutron-gamma discrimination

While the GRETA detectors and front-end electronics are custom engineered, the data acquisition system (DAQ) employs commercial laboratory equipment consisting of NIM and VME modules and a Windows XP computer running LabVIEW software. Key elements are a fast logic module to recognize coincident D1 and D2 CFD timing signals, a time-to-amplitude converter (TAC) to measure the time-of-flight (ToF) between the two detectors and peak sensing ADCs to convert the pulse heights, PSD and ToF for each registered coincidence. In the present DAQ configuration, all signals from D1 and D2 as well as the PSD and ToF are converted and recorded. Position of 
interaction in each detector is determined from maximum PMT signal for each event. This means that we know only that the photons interacted somewhere within the volume defined by the PMT locations. This limits the angular resolution to approximately $4^{\circ}$. Finer angular resolution $\left(\sim 1.5^{\circ}\right)$ can be achieved if all 15 (8 D1 +7 D2) PMT signals are used with appropriate additional calibration and location algorithms. This, in fact, is how COMPTEL operated in orbit. The next effort for this to be achieved is entirely in code additions and modifications.

Other than pointing the instrument, there is no adjustment to make. The main data selection to make (adjustment) is a selection on the measured energies. Energy bins of interest can be bracketed for further imaging information.

\section{PERFORMANCE DEMONSTRATION}

\section{User Interface}

A custom user interface has been developed to operate GRETA in real time mode (Figure 5.) The interface is written in LabVIEW 8.5 and allows for simultaneous source identification and location feedback. All data is subsequently stored to file for any post process analysis.
The user can choose to image multiple energy bands multiple source identifications. Currently an image plane is assigned for the appropriate source distance, but future versions will have the ability to "focus" on sources of interest for multiple fields of depth.

\section{Source Identification and Location}

A $750 \mu \mathrm{Ci}{ }^{137} \mathrm{Cs}$ source was used to demonstrate GRETA's ability to identify and locate the position of an unknown source. The instrument was operated in a normal fashion, i.e., data were collected with a display of energy and event circles in real time.

Each valid event produces an event circle that are projected on the source plane. The intersections of the circles are binned and the source location can be deduced by searching for the majority of intersections (Figures $6 \& 7$.) The source was first placed $2.89 \mathrm{~m}$ off the detector axis at a distance of $5 \mathrm{~m}$. The data shown in Figures 6 is for a 10 min run time. The source was then relocated to $5 \mathrm{~m}$ off-axis and run for the same $10 \mathrm{~min}$ interval. The plots indicate the shift in position that agrees with the true location of the source. The specific isotope of the source can be identified by using the recorded total energy spectrum, Figure 8.

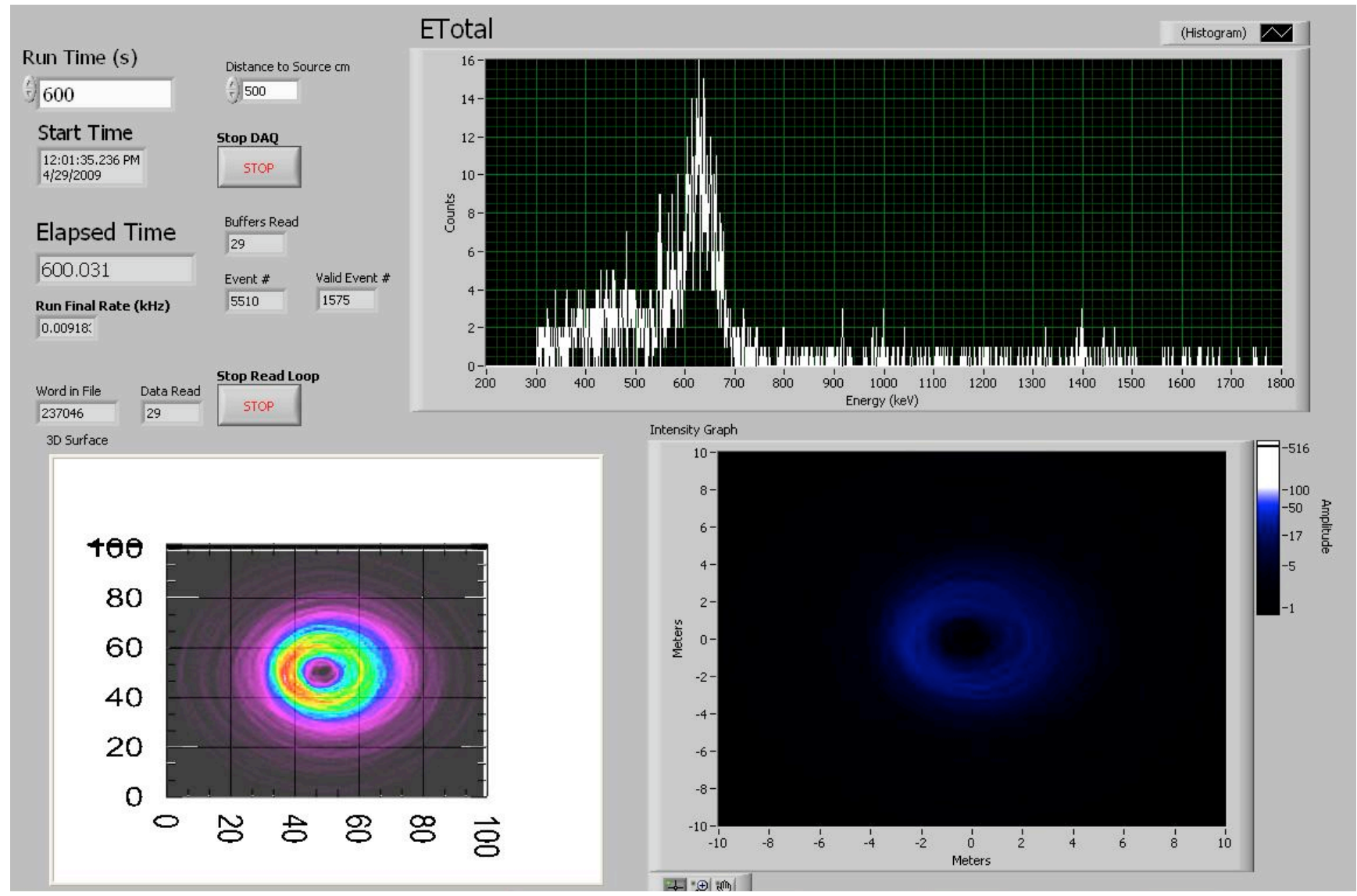

Figure 5 - Custom LabVIEW user interface showing $10 \mathrm{~min}$ data run of $750 \mu \mathrm{Ci}{ }^{137} \mathrm{Cs}$ source $2.89 \mathrm{~m}$ off-axis at a distance of $5 \mathrm{~m}$. 


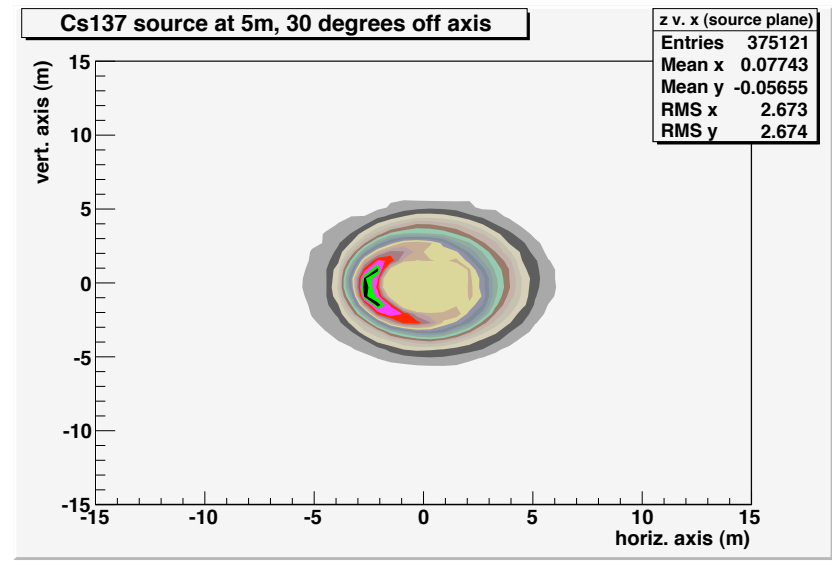

Figure $6-{ }^{137} \mathrm{Cs}(750 \mu \mathrm{Ci})$ image produced with the source $2.89 \mathrm{~m}$ off-axis at a distance of $5 \mathrm{~m}$.

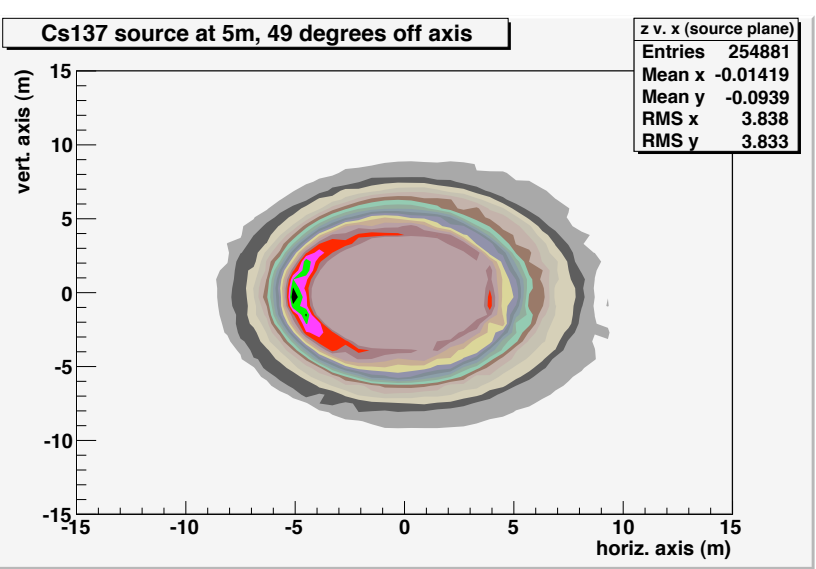

Figure $7-{ }^{137} \mathrm{Cs}(750 \mu \mathrm{Ci})$ image produced with the source $5 \mathrm{~m}$ off-axis at a distance of $5 \mathrm{~m}$.

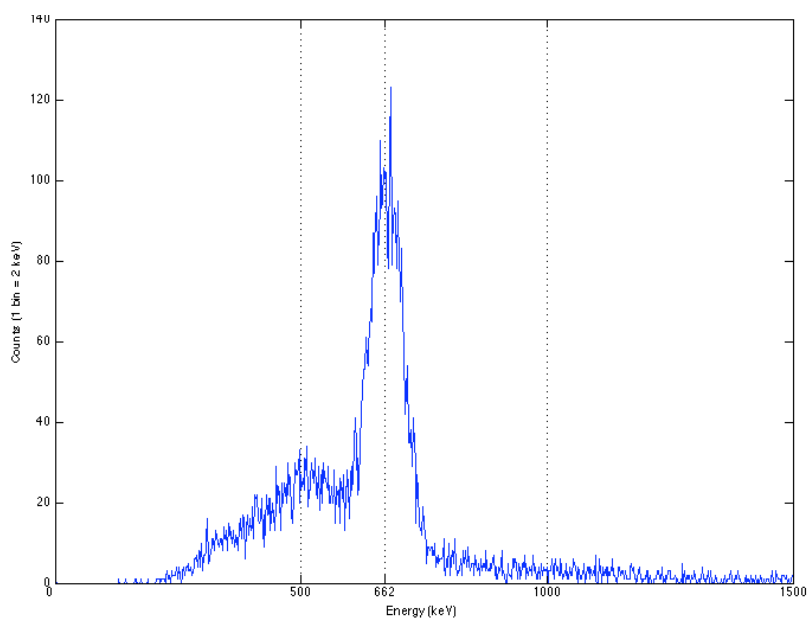

Figure 8 - ${ }^{137} \mathrm{Cs}(750 \mu \mathrm{Ci})$ spectrum measured with the source $2.89 \mathrm{~m}$ and $5 \mathrm{~m}$ off-axis at a distance of $5 \mathrm{~m}$.

\section{Detector Response}

A scan of the detector response as an $750 \mu \mathrm{Ci}{ }^{137} \mathrm{Cs}$ source was moved from within the field of view, on-axis, to outside the field of view is shown in Figure 9. The source was between 3 and $5 \mathrm{~m}$ from the instrument with two intervening interior walls. Presented are the count rates of double-scatter events as compared to single scatter events. The single scatter events are intended to simulate the performance of an omni-directional, perhaps hand held, instrument. The singles rate shows no significant modulation across the field of view, whereas the doublescatter rate in the $662 \mathrm{keV}$ photopeak with a preset field of view of $60^{\circ}$ degrees shows significant modulation as the source reaches the edge of the field of view. At the location of GRETA, the singles count rate is dominated by background and there is little change in the rate as the source is moved. However, the imaging properties of GRETA reveal the location of the source with respect to the instrument axis. The energy count spectrum of the ${ }^{137} \mathrm{Cs}$ source is shown in Figure 8. Although undetectable in the singles mode of the instrument, in the double-scatter mode,

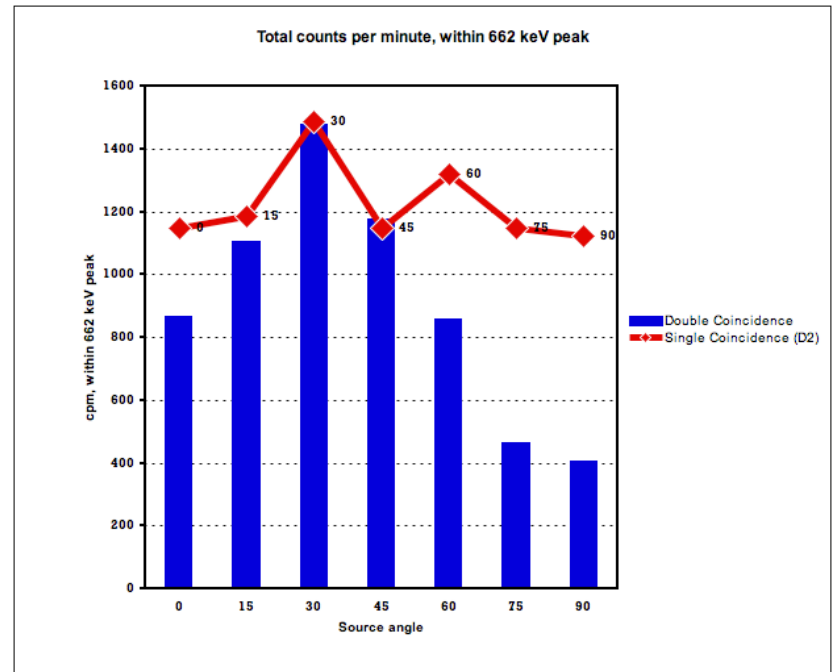

Figure $9-{ }^{137} \mathrm{Cs}(750 \mu \mathrm{Ci})$ image produced with the source 49 degrees off-axis at a distance of $5 \mathrm{~m}$.

the source reveals itself.

Comparable measurements were made using the ${ }^{137} \mathrm{Cs}$ and a lab survey meter. The meter is a calibrated Ludlum Geiger counter, model M-14C, with M-44-38 probe for $\gamma$-ray monitoring. With the meter positioned at D1 and no sources present in the lab a background rate of $\sim 0.05 \mathrm{mR} / \mathrm{hr}$ is measured. With the source at $3 \mathrm{~m}$ the rate increases to $\sim 0.10$ $\mathrm{mR} / \mathrm{hr}$, barely discernable. At a distance of $5 \mathrm{~m}$ there is no apparent change in measured rate.

\section{Multiple Sources}

A laboratory demonstration was conducted inside Morse Hall at the University of New Hampshire. The objective 
was to see if GRETA could detect the presence of two sources in its field of view while simultaneously imaging each respectively. The first source, an $750 \mu \mathrm{Ci}{ }^{137} \mathrm{Cs}$ source on the far side of two interior walls at a distance of $5 \mathrm{~m}$ and $5 \mathrm{~m}$ off-axis, and the second an $50 \mu \mathrm{Ci}{ }^{60} \mathrm{Co}$ source also at $5 \mathrm{~m}$ and $2.89 \mathrm{~m}$ off-axis. Data was taken for a $10 \mathrm{~min}$

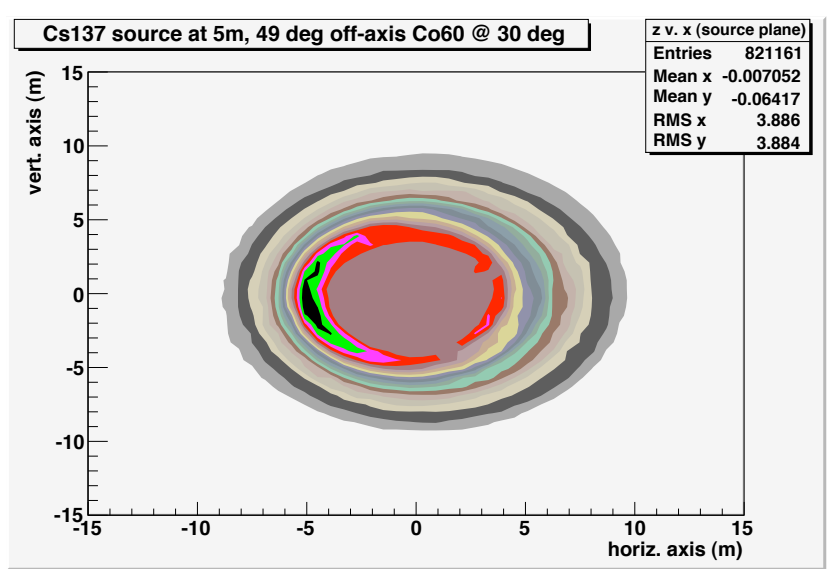

Figure $10-{ }^{137} \mathrm{Cs}(750 \mu \mathrm{Ci})$ image produced with the source $5 \mathrm{~m}$ off-axis at a distance of $5 \mathrm{~m}$.

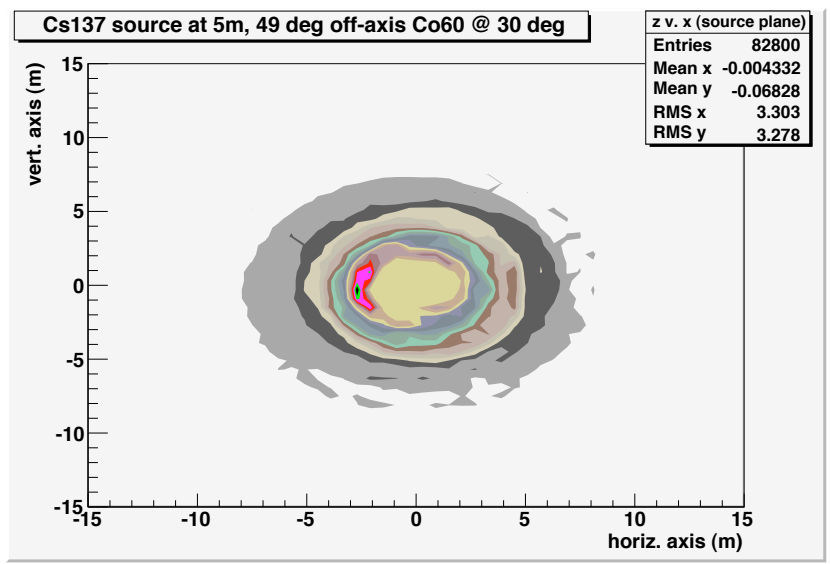

Figure $11-{ }^{60} \mathrm{Co}(50 \mu \mathrm{Ci})$ image produced with the source $2.89 \mathrm{~m}$ off-axis at a distance of $5 \mathrm{~m}$.

interval as in the previous runs. Again, the instrument was operated in normal fashion.

Figures 10 and 11 represent the imaging data obtained from the single data run. Source locations for the respective isotopes are correctly represented by the image plots. GRETA has the ability to clearly discriminate from the background multiple sources in multiple locations.

\section{CONCLUSIONS}

We describe the design and response of the GRETA Compton telescope. The instrument was configured to locate and identify unknown $\gamma$-ray sources with an $\sim 4^{\circ}$ angular resolution and $\sim 10 \%$ FWHM energy resolution.
Our tests have validated the design concept, demonstrating that it can locate a small ${ }^{137} \mathrm{Cs}$ at some distance in minimal time. With improvements in data acquisition and imaging algorithms, GRETA is capable of improvement better than $2^{\circ}$ as demonstrated on COMPTEL. Calculations done from the presented data indicate an effective area of $\sim .242 \mathrm{~cm}^{2}$ at $662 \mathrm{keV}$. We calculate that in order to obtain comparable image data for a $1 \mathrm{Ci}^{137} \mathrm{Cs}$ source at $50 \mathrm{~m}$ to be on the order of $70 \mathrm{~s}$.

We have defined and tested the design for a field deployable, passive detector system that can locate and identify RAM at large standoff distances. Future work includes comparison of laboratory measurements with Monte Carlo simulations, and more field measurements and demonstrations of the instrument.

\section{REFERENCES}

[1] James E. Doyle, Nuclear Safeguards, Security, and Nonproliferation, Achieving Security with Technology and Policy, New York: Elsevier, 2008

[2] Diehl et al., "Data Analysis of the COMPTEL Instrument on the NASA Gamma Ray Observatory," NASA. Goddard Space Flight Center, The Compton Observatory Science Workshop, 95-101 (1992).

[3] Schönfelder et al., "Instrument description and performance of the Imaging Gamma-Ray Telescope COMPTEL aboard the Compton Gamma-Ray Observatory," Astrophysical Journal Supplement Series, 86, 657-692 (1993).

[4] den Herder et al., "COMPTEL: instrument description and performance," NASA. Goddard Space Flight Center, The Compton Observatory Science Workshop, 85-94 (1992). 